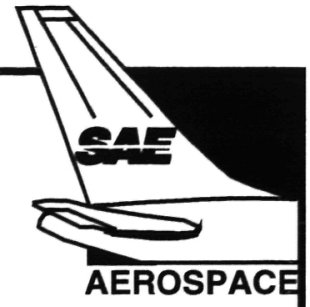

SAE 1999 - 01 - 5619

Advanced Aerodynamic Control Effectors

Richard M. Wood and Steven X. S. Bauer

NASA Langley Research Center

Hampton, Virginia 


\title{
Advanced Aerodynamic Control Effectors
}

\author{
Richard M. Wood and Steven X. S. Bauer \\ NASA Langley Research Center
}

\section{ABSTRACT}

A 1990 research program that focused on the development of advanced aerodynamic control effectors (AACE) for military aircraft has been reviewed and summarized. Data are presented for advanced planform, flow control, and surface contouring technologies. The data show significant increases in lift, reductions in drag, and increased control power, compared to typical aerodynamic designs. The results presented also highlighted the importance of planform selection in the design of a control effector suite. Planform data showed that dramatic increases in lift $(>25 \%)$ can be achieved with multiple wings and a sawtooth forebody. Passive porosity and micro drag generator control effector data showed control power levels exceeding that available from typical effectors (moving surfaces). Application of an advanced planform to a tailless concept showed benefits of similar magnitude as those observed in the generic studies.

\section{INTRODUCTION}

As we move into the new millennium, the technical and political challenges for the aerodynamic community are significant and continued success will require that all available information and knowledge be utilized to guide future aircraft development activity. This is especially true for the military community that must battle the challenges created by the diverse and dramatically changing threat environment. With this in mind, it is clear that we must review the technical accomplishments of the past before we attempt to create the future.

A review of the history of aircraft design (references 1 to 8) reveals that aerodynamic control effector technology has changed little since the early 1900s. Aircraft have tended to utilize "typical" suites of moving surface control effectors such as rudders, elevators, flaps, ailerons, etc. One exception to this trend is the use of thrust vectoring for control (i.e. AV8B and the F22).
Our present approach to aircraft design is highly influenced by our national economic policies. These influences have resulted in acquisition cost and operational cost of new airborne weapon systems to be the most critical elements in determining if a new system is developed. This change has placed an increased burden on aircraft designers to develop cost effective aircraft concepts that satisfy multi-disciplinary, multi-role, and multi-mission design challenges. Future aircraft designs must also have dramatic improvements in survivability to address the daily advancements in electronic sensor technology used to detect and track military aircraft. In addition to the need for survivability, there is also a need for low drag and the ability to be highly maneuverable.

This paper will review the results (references 9 to 12) of a study conducted at the NASA Langley Research Center from 1989 to 1992 to develop advanced aerodynamic control effector concepts required for new millennium air vehicles. In the late $1980 \mathrm{~s}$, it was recognized that to advance the state-of-the-art in military aircraft design, the development of the next generation of control effectors must address signature, weight, and maintainability issues while offering dramatic improvements in aerodynamic efficiency. As a result, the focus of this study was to develop control effector technologies consistent with the development of an aircraft that would not have gaps, cracks, steps, holes, etc. in all surfaces visible by advanced electromagnetic detection systems. The selected control effector technologies are required to satisfy projected survivability, drag, and agility requirements. The design philosophy and selected data for advanced aerodynamic control effector concepts consistent with this philosophy are presented in the following sections of this paper. Particular emphasis will be placed upon passive porosity technology, micro flowmanagement devices, and advanced planforms. 


\section{AACE PROGRAM}

The Advanced Aerodynamic Control Effector (AACE) program was an ad hoc activity created by a group of researchers at the NASA Langley Research Center. The team was formed in 1989 with a focus of developing "NEW" aerodynamic technologies in a multidisciplinary environment that supported the development of advanced military vehicles for the projected threat environment in 2010.

The goal of the AACE team was to create new aerodynamic technologies that allow for a dramatic improvement in both aerodynamic performance and survivability performance with respect to the levels available with existing technologies. An implied goal within the program was to create a significantly lighter and cost effective vehicle, compared to existing aircraft.

In pursuit of this goal, advanced three-dimensional shaping, advanced planforms, micro devices, passive pneumatics, and inflatables were identified as target technologies. From 1989 to 1992 , the activity executed more than 30 experimental test programs involving more than 60 models in 5 test facilities at the NASA Langley Research Center, references 9 and 12. The AACE activity consisted of approximately 5 work years of effort each year with a budget of approximately $\$ 200,000.00$ per year.

The following sections of this report will review the research conducted from 1989 to 1992.

\section{DESIGN PHILOSOPHY}

A typical design approach is to execute a hierarchical scheme in which identification, development, and implementation of control effectors is performed sequentially on a pre-designed vehicle with fixed planform. In contrast to a typical approach, the AACE activity employed a scheme in which all aerodynamic control effector technologies would be identified, developed, and implemented in parallel. This approach would allow each technology development activity to follow the path of the most significant technical challenge.

Another aspect to AACE was that the planform was recognized as being a primary contributor to control effector performance and the surface contouring was recognized as being secondary contributor to control effector performance. The recognition of these two points were critical to the success of the activity because it recognized that planform and surface contour are responsible for much more than creating lift and establishing stability levels at low lift conditions. The approach used in AACE required the planform and surface contouring to establish the aerodynamic performance, controllability potential, and survivability potential of the vehicle over a broad range of angle-ofattack and mach number. These characteristics were especially important when considering new classes of control effectors. It was thought that traditional planforms and surface contours might not provide the threedimensional loadings required to optimize the performance of the advanced aerodynamic control effectors.

The notion of moving large or even small sensor-visible elements of the aircraft parent surface (i.e., external and internal surfaces) to create control power is counter to the design constraints imposed by reduced cost, improved survivability, and low drag. In a first order analysis, the purchase and operational costs of an aircraft are proportional to the number of parts of an aircraft. Survivability is proportional to the number and types of physical breaks and curvature breaks in the sensorvisible parent surfaces and drag is proportional to the number of breaks in the flow-contact surfaces. To address these issues, the AACE activity focused on eliminating breaks in the parent surface and/or eliminating moving control effectors in order to reduce the number of parts and reduce actuation requirements; thus, decrease cost and drag and increase survivability.

\section{Design Space}

In conceptualizing the design philosophy, a design space was defined that would allow significant intellectual freedom while ensuring resulting technologies were relevant. As noted earlier, specific design goals were not identified in the AACE program, however, a set of objectives/constraints were identified up front, and these objectives/constraints were used to focus the team's efforts. The AACE objectives are listed below:

- Balanced signature

- High lift at low angle of attack $(\mathrm{M}<.3)$

- Maneuverability to 70 degrees angle-of-attack $(M<.9)$

- Dry supercruise $(\mathrm{M}<1.8)$

- $20 \%$ increase in usable volume

These objectives clearly show the broad range of technical challenges presented to the team. However, to place these objectives into perspective and to complete the design space definition a set of design constraints were imposed on the AACE concepts. . These constraints are listed below.

- Linear and aligned planform edges

- Planar planform perimeter 
- Minimize planform breaks

- No sensor-visible breaks in the parent surfaces

- No movement of the sensor-visible parent surfaces

- $2^{\text {nd }}$ derivative continuous sensor-visible surfaces

These constraints suggest that the chosen design space resides outside the realm of solutions recognized in the late 1980s.

\section{Design Strategy}

To meet the challenges described above; a design strategy was developed which assumed that success could only be achieved when approached from the most challenging direction. A graphical depiction of this point is presented in figure 1. The figure shows the variation in aircraft performance and aircraft weight due to design angle-of-attack (lift) for an existing design approach and for the AACE design strategy, in which the dominant design condition is the high angle-of-attack point. The present strategy assumes that it is easier to achieve high levels of off-design performance if you focus your design efforts on the most challenging design point and thus allow the most benign design point to be viewed as "offdesign".

A major concern of the activity was to reduce the influence of historical, but unproven, constraints and assumptions which tend to dominate many existing design activities. An example of an unproven historical aerodynamic assumption would be that the minimum drag boundary is defined by linear theory with $100 \%$ leading edge thrust, reference 13. Another assumption is that wing leading-edge bluntness increases wave drag at supersonic speeds, reference 13.

The final strategy employed by the team was to explore the use of interfering aerodynamic flow fields and electromagnetic fields to improve the aerodynamic performance and electromagnetic performance, respectively, of candidate technologies. In the aerodynamic arena, the effort focused on wake interaction studies, fuselage upwash (reference 14), base-drag management, and vortex management.

\section{AACE TECHNOLOGIES}

A list of the technologies included in the AACE activity are presented in table 1. In general, these technologies can be divided into three groups: planforms, flow control, and surface contouring. All experimental tests of the aerodynamic technologies were conducted on generic models in order to isolate the particular aerodynamic and flow control effect.
Noted in the table are the technologies for which experimental data have been obtained. Note that data are available for all technologies listed with the exception of Stagnation Point Control, listed under surface contouring, and Adapting/Flexible Surface, listed under both surface contouring and flow control. Both of these technologies were identified at the end of the program and as a result were not fully investigated.

Selected results from the planform group, flow control group, and surface contouring group will be presented in the following sections of the paper.

\section{Planform Technologies}

As mentioned previously, the planform was viewed as a primary contributor to control effector performance within the AACE activity. In particular, the activity focused on the development of planform concepts that would provide significant increases in lift and linear stability characteristics for angles-of-attack up to $70^{\circ}$. A total of 21 planforms were investigated in the activity. All of the planforms were a variation from the 6 planforms depicted in figure 2. As shown in figure 2, the planform types were a diamond (baseline configuration), twin-body, sawtooth forebody, twin-wing, cut-out wing, and joined wing. All planform models tested were constructed as flat plates with sharp edges. Tests were conducted at a Mach number of 0.17 . The data obtained for each planform was reduced with a reference area equal to the total planform area to ensure that differences in lifting surface area did not bias the results. Note that the use of the total planform area as the reference area will result in aerodynamic coefficients that are of lower value than those typically observed for configurations with similar planforms.

The twin-body and sawtooth forebody concepts were developed to investigate vortex flow interaction between the fuselage and wing structures as well as fuselage upwash (reference 14) in order to increase lift and to eliminate large nonlinear stability characteristics at moderate to high angles-of-attack. Another benefit of the twin-body concept is the ability to reduce drag at supersonic speeds through positive near-field interference (references 15 to 17). The notion behind the twin wing, cut-out wing, and joined wing planforms is to create aircraft designs with multiple primary lifting surfaces such that each surface can be turned off and on (i.e., loading increased, decreased, or eliminated) as required to create the desired flight characteristics. It was hypothesized that independent control of each primary lifting surface could increase lift and also allow control of the aircraft stability level at all angles-of-attack.

The longitudinal aerodynamic data (reference 9) obtained for all planforms showed that the twin wing and sawtooth forebody provided the largest increase in lift 
and significant improvements in longitudinal stability characteristics compared to the baseline geometry. Lift characteristics for the sawtooth forebody are presented in figure 3 and twin-wing results are presented in figure 4.

The data of figure 3 show that the sawtooth forebody concept provides a significant increase in lift for all angles-of-attack greater than $10^{\circ}$. The data also show that the sawtooth forebody concept provides a $25 \%$ increase in maximum lift over the baseline planform. Note, the magnitude of these benefits is depressed by the use of the total planform area as the reference area for data reduction. The benefit achieved by the sawtooth forebody concept results from both vortex lift acting on the sawteeth as well as the parent wing and an interfering flow field emanating from the sawteeth onto the parent wing. The interfering flow field is characterized by a downwash field that acts on the parent wing. This allows the parent wing to operate at an effective lower angle of attack thereby reducing separation. It is important to note that the sawtooth forebody concept has been shown to be applicable to other planform shapes (reference 9).

The data of figure 4 show that the twin wing concept also provides a significant increase in lift starting at $20^{\circ}$ angleof-attack and extending to $70^{\circ}$ angle-of-attack. The data show that the twin wing concept also provides a $25 \%$ increase in maximum lift over the baseline planform. It is conjectured that the benefit achieved by the twin wing concept results from reduced flow separation on the aft wing due to a strong downwash field from the forward wing. This downwash allows the aft wing to operate at an effective lower angle of attack thereby reducing separation. It is important to note that the multiple wing concept has been shown to be applicable to other planform shapes (reference 9).

These data show that the high-lift performance of aircraft can be greatly enhanced through the use of multiple primary lifting surfaces and interfering flow fields. Additional results will be shown to document the high speed performance of these planforms.

\section{Flow Control Technologies}

A total of five different flow control technologies were identified in the AACE program and four were investigated. Based upon initial results, the majority of the effort was directed towards passive porosity technology and inflatable/deployable micro bumps. A brief overview of each of these two technologies is presented below.

\section{Passive Porosity Technology}

The passive porosity technology has been extensively studied both experimentally and computationally as a means to control shock/boundary layer interaction, see references 10,18 to 21 . The focus of passive porosity in the AACE activity was for both local boundary layer management and global application of passive porosity on an aerodynamic vehicle to control the forces and moments of the vehicle.

Passive porosity is designed to modify and control the pressure loading acting on a surface The passive porosity concept consists of a porous outer surface and a solid inner surface. The volume between the outer and inner surfaces form an open plenum that is filled with the same fluid which is flowing over the exterior surface of the porous skin, see figure 5 . The effectiveness of the concept is dependent upon the ability of the system to allow unrestricted communication between large pressure differences on the external surface (high permeability).

A representative application of a passive porosity flow control effector is depicted in figure 6 along with the equivalent typical control effector (trailing edge flap). It is conjectured that passive porosity control effectors provide improved aerodynamic benefit, compared to typical effectors, because the change in force generated by the passive porosity device acts perpendicular to the vehicles principal plane and as a result would have a greater moment arm. It is also argued that the passive porosity effector would be more effective because it produces a linearly varying control force with increasing lift whereas the typical effector control force nonlinearly decreases with increasing lift.

The passive porosity effector would be configured with a means to control the permeability of the passive porosity system. The means to activate and deactivate the passive porosity system may be accomplished by reducing the permeability of the porous surface or by reducing the permeability of the plenum. The porous surface permeability may be controlled by restricting the size of or closing the passages through the porous surface with a smart skin technology or by covering the internal surface of the porous surface with a non porous surface or low permeability surface. The permeability of the passive porosity system may also be controlled by changing the plenum characteristics.

Passive porosity control effector development was performed on a series of wing and fuselage models. The two wing models were a $65^{\circ}$ Delta wing and a high aspect 
ratio wing with a $\mathrm{GA}(\mathrm{W})-1$ airfoil (reference 21 ). Both wing modeis were porous over their complete surface area in order to maximize the flexibility of the models. In addition fuselage studies were conducted on a 2.5 and 5.0 caliber, tangent-ogive models. The porosity for all passive porosity models was nominally $22 \%$ with hole diameters of approximately. 020 inch. The focus of the wing models was to investigate roll and pitch control effectiveness and the forebody models were used to investigate yaw control. Applying tape over the porous surface to create a solid surface varied the extent of the porous surface on all models.

Representative passive porosity control effector results at a Mach number of 0.17 for the $65^{\circ}$ Delta wing model are presented in figures 7 and 8 . Also shown in figure 7 are representative control effectiveness data for the F/A18 aircraft (references 22 and 23). Because a control effectors ability to provide pitch control is highly dependent on vehicle layout it was decided that the potential of passive porosity to provide pitch control would be evaluated by investigating the change in lift coefficient, see figure 8 .

Roll control for various extents of tip porosity is shown in figure 7 and changes in lift coefficient for various extents of trailing-edge porosity are shown in figure 8 . The data are for configurations with porosity applied to both the upper and lower surface. This application of porosity allows the passive porosity system to eliminate (dump) lift on a particular region of a wing. The data of figures 7 and 8 clearly show that significant control authority is available with this technology, at moderate angles-of-attack. A comparison of the passive porosity results with those for the conventional aerodynamic control effector show that the passive porosity device is more effective for anglesof-attack greater than $10^{\circ}$.

The failure of the passive porosity device to generate control forces at low lift is due to the symmetric loading on the airfoil at these conditions. As mentioned previously, passive porosity effectiveness is a function of the pressure differential on the surface. Geometries studied in the AACE activity were symmetric, and thus, they would have negligible pressure gradients at zero lift. This observation supports the need to view the planform and surface contour as important contributors to control effectiveness. To maximize the effectiveness of the passive porosity control effector, large pressure gradients must exist on the surface of the wing or fuselage at all angles of attack. To create this environment may require new families of planforms and surface contours.

Figure 9 presents side force data for a porous 5.0 caliber, tangent-ogive forebody model with various circumferential extents of porosity, a porous forebody with chine, and the F/A-18 HARV (reference 24) with actuated strakes. Also shown on the figure is the available side force from the F/A-18 vertical tail (reference $25)$. In the region where asymmetric vortex shedding typically occurs $\left(\alpha>20^{\circ}\right)$, the application of $360^{\circ}$ of porosity eliminates the asymmetric vortex loading. Application of porosity to the left side of the forebody allows for maximum control of the side force. The addition of a chine to the same model increases the side force contribution of passive porosity. As observed with the wing data shown in figure 7, a comparison of the generated forebody forces shows significant increases at angles-of-attack greater than $25^{\circ}$ over that available with more traditional movable control effectors, a vertical tail or actuated forebody strakes.

\section{Micro-Drag Generators (MDGs)}

The Micro-flow-management devices that were investigated include micro-drag bumps, spoilers, and splitter plates. These concepts modify the boundary layer in order to promote or inhibit flow separation that in turn creates large changes in the aircraft forces and moments. These concepts would work in concert with other flow control and surface contouring technology to enhance the naturally occurring pressure field over the configuration in order to maximize the desired aerodynamic force.

The Micro Drag Generator concept (reference 11) uses small deployable devices referred to as MDGs that individually generate small amounts of drag, but when deployed in large numbers can generate substantial amounts of drag. The micro-drag generators (MDGs) may be thought of as miniature spoilers or speed brakes. During normal operation of the vehicle (e.g., during cruise), the devices would not be extended into the flowfield and would not increase the drag of the vehicle.

MDGs are designed to force the flow on a vehicle to separate on the aft-facing side of the device and to reattach before reaching the next device, see figures 10 and 11. Note that the MDG devices tested were sized for wind-tunnel conditions (i.e. thicker boundary layer in the wind tunnel than would be seen in flight for the same wing chord) to ensure the concept was properly evaluated. The MDG concept allows substantial amounts of drag to be generated with a simple system of small devices. The drag generated by a system of MDGs is expected to be equivalent to that generated from a single device with the same projected area as the sum of all the MDG projected areas. Because MDGs are very nonintrusive, the application of such devices on military aircraft as a control effector is quite attractive (reduced weight and complexity)

MDGs were experimentally investigated on the right hand side of a high aspect ratio wing with the $G A(W)-1$ airfoil, see figure 11, and also on a family of axisymmetric bodies. The wind-tunnel data indicated that the 
deployment of MDGs on a wing can increase the drag by as much as $400 \%$ (medium density, large drag plates), see figure 12 . The $400 \%$ increase in wing drag equates to a change in drag coefficient, between the clean wing and the wing with MDG's, that varies from 0.04 at zero-lift to 0.11 at a lift coefficient of 0.5 . The magnitude of this asymmetric force is equivalent to the side force generated by the F/A-18 actuated forebody strakes (references 22 and 23). Both concepts can generate useful amounts of yawing moments for control. A review of the data contained in reference 11 shows that MDG's can also be used to reduce lift.

These results indicate that by asymmetrically deploying MDGs (only on one wing panel) to an aircraft substantial amounts of control effectiveness (both rolling- and yawing-moment coefficients) may be generated. Therefore, MDGs appear to be an effective concept for decelerating or controlling a vehicle.

\section{Surface Contouring Technologies}

The final area of aerodynamic technologies to be discussed are those related to surface contouring. The notion that surface contouring has a significant impact on control effector performance is undoubtedly quite unusual, however, it is critical if we are to maximize the benefit of this suite of new technologies. Surface contouring creates the optimum surface to receive the naturally occurring loading generated by the planform and works in parallel with flow control effectors to increase or decrease the loading. Surface contouring technologies must produce the loadings required by the flow control effectors over a broad flight envelope as well as provide low drag. Note, surface contouring is not performed to create a predetermined loading or a predetermined aerodynamic characteristic.

The surface contouring technologies that have been investigated vary significantly in their approach used to improve the aerodynamic and vehicle performance. The Natural Flow, Multi-Body, and Blunt Trailing Edge technologies create the optimum three dimensional shape over which the naturally occurring flow field, and resulting pressures, may act. Passive Porosity and Adapting/Flexible Surface are used to either fluidically or physically change the external surface to match the flow field in an effort to reduce or eliminate separation. Adapting/Flexible Surface technology was viewed as the lowest ranking technology because of its complexity and because it violated the design philosophy of eliminating all moving surfaces. The highest-ranking surface contouring technology was the Natural Flow Wing (NFW) design approach(reference 12 and 26) because of its robustness and flexibility. The remainder of this section will highlight this technology.
The natural flow surface contouring design approach is based upon the observation that the characteristic surface loading of an aircraft is primarily determined by the vehicles planform and the global loading pattern varies little with changes in Mach number and angle-ofattack (reference 13). This observation allows the designer to create the optimum three-dimensional surface for the vehicle planform for the characteristic surface loading to act. This observation and design approach is equally applicable to planar shapes or fuciform shapes.

The application of the Natural Flow design concept to a delta wing is graphically depicted in figure 13 . For a typical delta wing the application of thickness results in a wing geometry that is conical about the wing tip. However experimental data (reference 13) show that the flow is conical about the wing apex. The figure shows that the flow over the upper surface of a swept wing is characterized by an expansion over the leading edge that is followed by a recompression to a more positive pressure as the flow moves inboard and aft. If the upper surface of the standard wing is divided into four quadrants, defined by the intersection of the airfoil maximum half-thickness line (crest line) and the crossflow recompression line, two favorable and two unfavorable regions are identified. The two unfavorable regions, which contribute to drag, are the inboard forward region $(A)$ and the outboard aft-region $(C)$ of the wing. Region $A$ has high pressures acting on a forward facing surface and region $C$ has low pressures acting on an aft-facing surface. Regions $B$ and $D$ have pressure fields, which combine favorably with the local surface, slope to reduce drag. The Natural Flow design approach is to reduce the size of regions $A$ and $C$ and increase the size of regions $B$ and $D$.

The Natural Flow wing lower surface design concept is also shown in figure 13. The sketch on the right side of figure 13 show that the lower surface pressure loading is characterized by high pressure over the complete lower surface. The combination of this high pressure and the forward facing surface of region A combine to increase drag and the high pressure combined with the aft facing surface of region $D$ reduces drag. As discussed above the design approach is to reduce the size of region $A$ and increase the size of region $D$.

To investigate this technology, an aerodynamic design study and experimental validation was conducted. The . design activity was performed on a $65^{\circ}$ delta wing for a Mach number of 1.62 and a design lift coefficient of 0.3 . A photograph of the natural flow designed wing is shown in figure 14. The design has negligible surface curvature (i.e., no inflection points) and all planform edges reside in a single plane. The natural flow design wing, a baseline 
symmetric wing with a NACA 64a-series airfoil, and a symmetric near-conical wing (reference 26) with a variable-thickness, NACA 64a-series airfoil were tested over a range of Mach number range of 1.6 to 2.0 and angles-of-attack from $-4^{\circ}$ to $20^{\circ}$.

Surface oil flow photographs are presented in figure 15 for the three wings and longitudinal aerodynamic characteristics of the three wings are shown in figure 16 . The oil flow photographs of figure 15 are for a lift coefficient of 0.3 and show that all three wings have nearly identical surface flow characteristics despite having large differences in $L / D$, see figure 16. Note that the dark regions at the tip of each wing is a result of lighting and does not reflect a change in flow characteristics. The data of figure 16 clearly show that the natural flow wing provides the same performance as the symmetric wings at zero lift and outperforms the symmetric wings at lifting conditions. In general, the natural flow design provides approximately a 10\% improvement in aerodynamic performance with a nonsymmetric and planar geometry.

\section{ADVANCED AIRCRAFT CONCEPT}

The results presented show that AACE technologies provide increased lift, reduced drag, and increased control authority, compared to typical aircraft concepts. The data also indicate that these results are consistent with a fixed geometry, rigid vehicle with minimal moving surfaces. To further refine the concepts, an investigation of the aerodynamics of a representative advanced vehicle configured with AACE technologies was conducted.

The AACE advanced concept was developed by rewinging an existing advanced tailless concept. The baseline vehicle consisted of a chined fuselage and a $30^{\circ}$ swept trapezoidal/diamond wing. The redesign effort focused on replacing the single $30^{\circ}$ diamond wing with twin $30^{\circ}$ diamond wings as depicted in figure 17. A primary design consideration was the minimization of supersonic cruise drag for a Mach number of 1.8. The redesigned configuration was highly constrained by the wing/fuselage attachment points for the existing highspeed wind tunnel model. The resultant twin wing design was constrained to have the same exposed wing area and the same wing volume as that of the baseline wing. These two requirements in combination with the wing/body attachment restrictions penalized the twin wing design activity. A photograph of the AACE advanced twin wing concept installed in the NASA Langley Research Center Unitary Plan Wind Tunnel is shown in figure 18.

Selected experimental results from the supersonic test program are presented in figures 19 and 20. Figure 19 shows the variation in zero-lift drag with Mach number for the baseline and twin wing concepts. The drag data show that the two concepts are similar with the twin wing concept having approximately 2 to $4 \%$ greater drag than the baseline. These results are very encouraging when considering the fact that the baseline configuration was area ruled for the design point and the twin wing design activity was limited by the existing hardware.

Presented in figure 20 is a summary of the lift characteristics over the Mach range of 1.6 to 2.2 for the baseline and twin wing concepts. The data clearly show that the lift curve slope for the twin wing is approximately $14 \%$ greater than the lift curve slope for the baseline geometry. Note that these data are consistent with the results of the planform study discussed previously. Analysis of the results of figures 19 and 20 show that the increased lifting efficiency of the twin-wing concept more than compensated for the increase in zero-lift drag to produce a vehicle with improved aerodynamic efficiency.

\section{CONCLUDING REMARKS}

A 1990 research program that focused on the development of advanced aerodynamic technologies (AACE) for military aircraft has been reviewed. A new class of advanced aerodynamic control effector technologies have been presented and discussed. These new technologies were developed to meet the following requirements; invisible to electromagnetic energy, aerodynamically robust, and light weight. The technologies must be aerodynamically and mechanically simple and they must minimize the power/energy requirements for operation.

Data presented for planform, flow control, and surface contouring technologies have shown significant increases in lift, reduced drag, and increased control power, relative to typical aerodynamic designs. The results presented also highlighted the importance of planform in the design of a control effector suite. The planform data showed that dramatic increases in lift (> $25 \%$ ) can be achieved with multiple wings and the sawtooth forebody. Passive porosity and micro-drag generator control effector data showed control power levels exceeding that available from typical effectors (moving surfaces) at moderate to high angles-of-attack. Application of an advanced planform to an tailless concept showed benefits of similar magnitude as that observed in generic planar configuration studies.

\section{Acknowledgments}

The authors would like to acknowledge the outstanding contributions of Brian McGrath of Johns Hopkins Applied Physics Laboratory, Greg Gatlin and Ken Goodrich of National Aeronautics and Space Agency, and Pat O'Neil and Garry Billman of Boeing Airplane Company. 


\section{REFERENCES}

1. Rolfe, D., Dawydoff, A., Winter, W., Byshyn, W., and Clark, H; Airplanes of the World, 1490 to 1969. Simon and Schuster. 1969.

2. Taylor, M. and Mondey, D.; Guiness Book of Aircraft Facts and Feats. Guiness. 1984.

3. Wooldridge, E. T.; Winged Wonders The Story of the Flying Wings. National Air and Space Museum. 1983.

4. Myhra, D.; The Horten Brothers and Their All-Wing Aircraft. Schiffer Publishing. 1998

5. Gibbs-Smith, C. H.; Aviation An Historical Survey from its Origins to the end of World War II.1985.

6. Anderson, F.; Northrop: An Aeronautical History. Northrop Corporation, Los Angeles, 1976.

7. Bowers, P. M.; Unconventional Aircraft. TAB Books, Blue Ridge Summit, PA. 1984.

8. Chambers, J. R.; High-Angle-of-Attack TechnologyProgress and Challenges. High Angle-of-Attack Technology, Volume I. NASA CP-3149, Part 1,1992 .

9. Gatlin, G. M. and McGrath, B. E.; Low-Speed Longitudinal Aerodynamic Characteristics Through Poststall for Twenty-One Novel Planform Shapes. NASA TP 3503. August 1995..

10. Wood, R. M., Banks, D. W., and Bauer, S. X. S.; Assessment of Passive Porosity with Free and Fixed Separation on a Tangent Ogive Forebody. AIAA-924494, 1992.

11. Bauer, S. X. S.; An Aerodynamic Assessment of Micro-Drag Generators (MDGs). 16 ${ }^{\text {th }}$ AIAA Applied Aerodynamics Conference. AIAA-98-2621.

12. Wood, R. M. and Bauer, S. X. S; The Natural Flow Wing Design Concept. NASATP 3193. May 1992.

13. Wood, R. M.; Supersonic Aerodynamics of Delta Wings. NASA TP 2771, 1988.

14. Wood, R. M. and Miller, D. S.; Effect of Fuselage Upwash on the Supersonic Longitudinal Aerodynamic Characterisitics of Two Fighter Configurations. NASA TP-2330, 1984.
15. Wood, R. M., Miller, D. S., and Brentner, K. S.; Theoretical and Experimental Investigation ọ Supersonic Aerodynamic Characteristics of a Twin Fuselage Concept. NASA TP-2184, 1983.

16. Wood, R. M., Rose, O. J., McMillin, S. N.; Effects of Body Cross-Sectional Shape on the Supersonic Aerodynamics of Multibody Configurations. NASA TP-2587, 1986.

17. McMillin, S. N. and Wood, R.M.; Planform Effects on the Supersonic Aerodynamics of Multibody Configurations. NASATP-2762, 1987.

18. Bauer, S. X. S. and Hemsch, M. J.: Alleviation of Side Force on Tangent Ogive Forebodies Using Passive Porosity, Proposed AIAA Paper 10th Applied Aerodynamics Conference, June 1992.

19. Wilcox, F. J.: Experimental Investigation of the Effects of a Porous Floor on Cavity Flow Fields at Supersonic Speeds. NASA TP 3032, 1990.

20. Nagamatsu, H. T. and Orozco,R. D.: Porosity Effect on Supercritical Airfoil Drag Reduction by Shock Wave Boundary Layer Control. AIAA-4-1682.

21. McGhee, R. J. and Beasley, W. D.: Low-Speed Aerodynamic Characteristics of a 17-Percent -Thick Airfoil Section Designed for General Aviation Applications. NASA TN D-7428. December 1973.

22. Erickson, G. E.;Wind Tunnel Investigation of Vortex Flows on F/A-18 Configuration at Subsonic Through Transonic Speeds. NASA TP-3111, 1991.

23. Erickson, G. E. and Murri, D. G.; Wind Tunnel Investigations of Forebody Strakes for Yaw Control on F/A-18 Model at Subsonic and Transonic Speeds. NASA TP-3360, 1993.

24. Regenie, V., Gatlin, D., Kempel, R., and Matheny, N.; The F-18 High Alpha Research Vehicle: A High Angle-of-Attack Testbed Aircraft. NASA TM-104253, 1992.

25. Buttrill, C. S.; Arbuckle, P. D.; and Hoffler, K. D.: Simulation Model of a Twin Tail High Performance Airplane. NASA TM-107601, 1992.

26. Bauer,X. S. X., Wood, R. M., and Brown, S. M.; A Natural Flow Wing Design Employing 3-D Nonlinear Analysis Applied at Supersonic Speeds. AIAA-892167, 1989. 


\section{DEFINITIONS, ACRONYMS, ABBREVIATIONS}

AACE advanced aerodynamic control effectors

$C_{D} \cdot \quad$ drag coefficient

$\mathrm{C}_{\mathrm{D}, 0} \quad$ zero-lift drag coefficient

$C_{L} \quad$ lift coefficient

-. $C_{L, \alpha} \quad$ lift curve slope

$\mathrm{C}_{\mathrm{Y}} \quad$ side force coefficient

$\Delta \mathrm{C}_{\mathrm{L}} \quad$ change in lift coefficient

$\Delta C_{\mid} \quad$ change in rolling moment coefficient

D hole diameter, inches

h plenum depth, inches

L hole spacing, inches

L/D lift to drag ratio

M mach number

MDG micro drag generators

NASA National Aeronautics and Space Administration

$P_{1} \quad$ external pressure, psi

$P_{2} \quad$ external pressure, $p s i$

t skin thickness, inches

TE trailing edge

$v_{e} \quad$ hole exit velocity

$\alpha \quad$ angle-of-attack, degrees

$\delta \quad$ boundary layer thickness, inches

- PLANFORMS

Multi-body*

- Serrated Body*

- Joined Wing*

- Multiple Wings*

- Cut-out Wings*

- SURFACE CONTOURING

- Natural Flow Design

- Multi-Body Design*

- Blunt Trailing Edge*

- Passive Porosity*

- Stagnation Point Control

- Adapting/Flexible Surface

FLOW CONTROL EFFECTORS

- Passive Porosity*

- Separation Control Plates*

- Inflatable Micro Bumps*

Adapting/Flexible Surface

- Boundary Layer Control*

* data available

Table 1. Candidate AACE technologies.

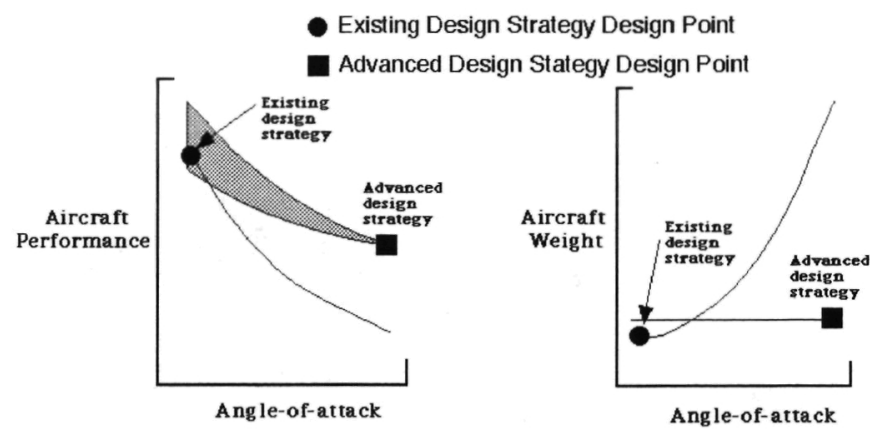

Figure 1. Graphic depicting AACE design strategy.

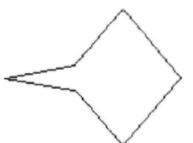

Diamond (baseline)

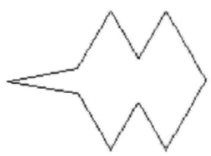

Twin Wing

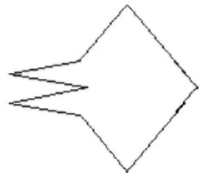

Twin Body

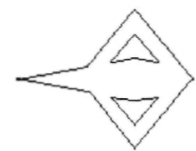

Cut-out

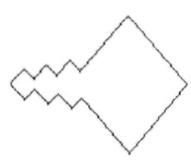

Sawtooth

Figure 2. Sketch of the six classes of planforms investigated in the AACE activity. 
$40^{\circ}$ Diamond and $40^{\circ}$ Sawtooth Diamond

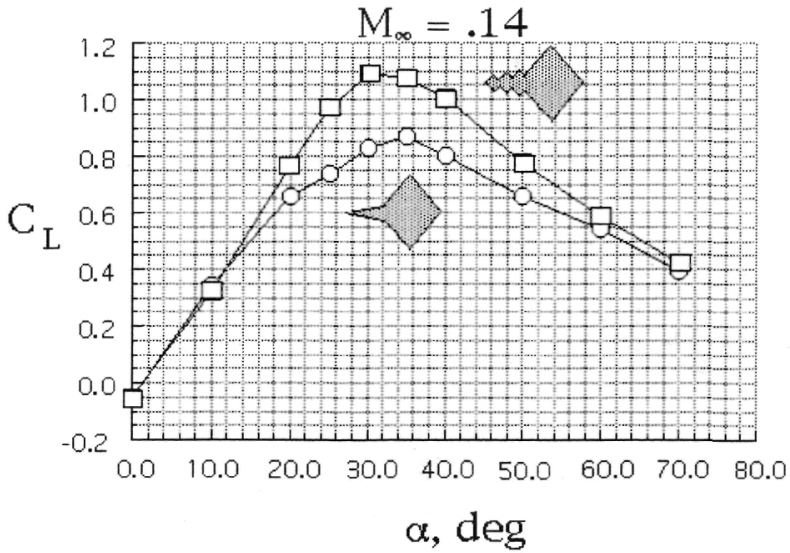

Figure 3. Plot of lift against angle-of-attack for the $40^{\circ}$ diamond planform and the $40^{\circ}$ sawtooth planform.

\section{$30^{\circ}$ Diamond and $30^{\circ}$ Twin Diamond}

$$
\mathrm{M}_{\infty}=.14
$$

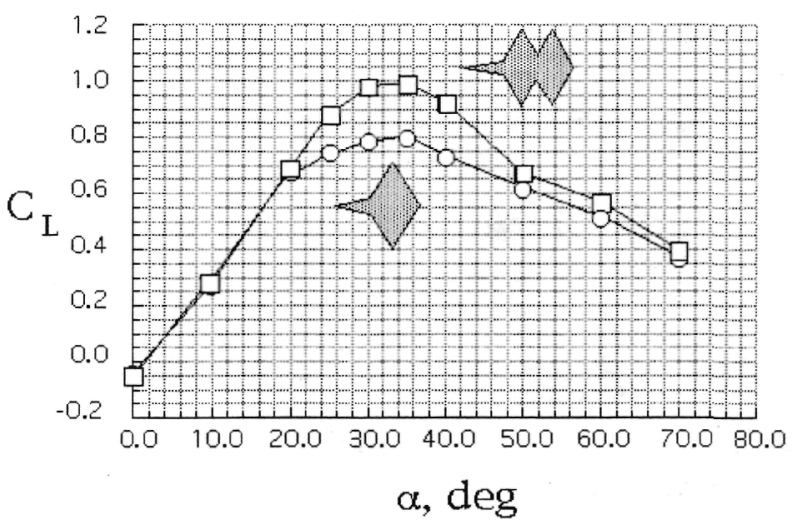

Figure 4. Plot of lift against angle-of-attack for the $30^{\circ}$ diamond planform and the $30^{\circ}$ twin diamond planform.

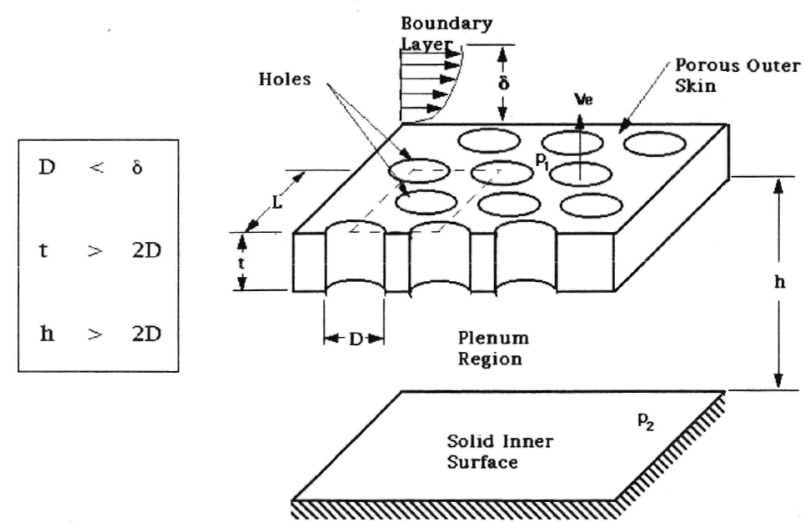

Figure 5. Schematic of passive porosity system.
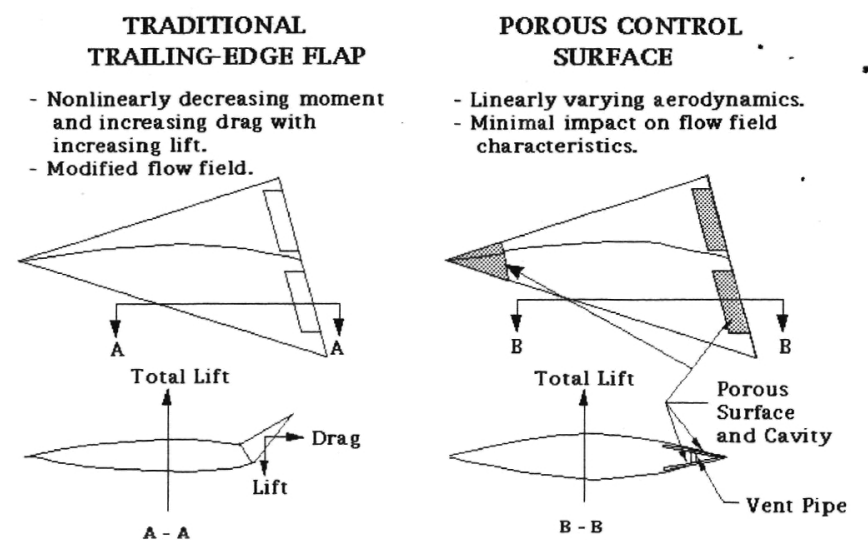

Figure 6. Sketch comparing traditional control effector with passive porosity control effector.

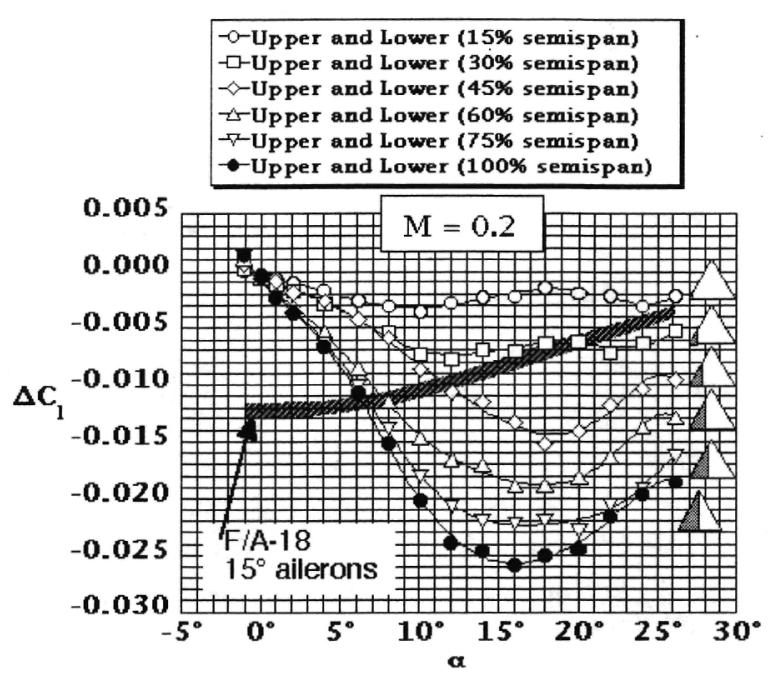

Figure 7. Plot of rolling moment increment for various passive porosity applications to the tip of the $55^{\circ}$ delta wing.

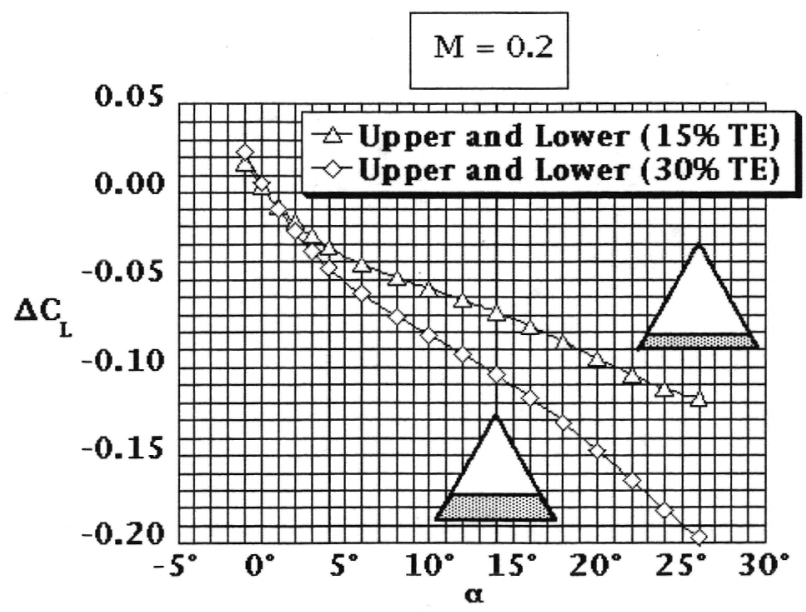

Figure 8. Plot of the lift coefficient increment for various passive porosity applications to the trailing edge of a $55^{\circ}$ delta wing. 


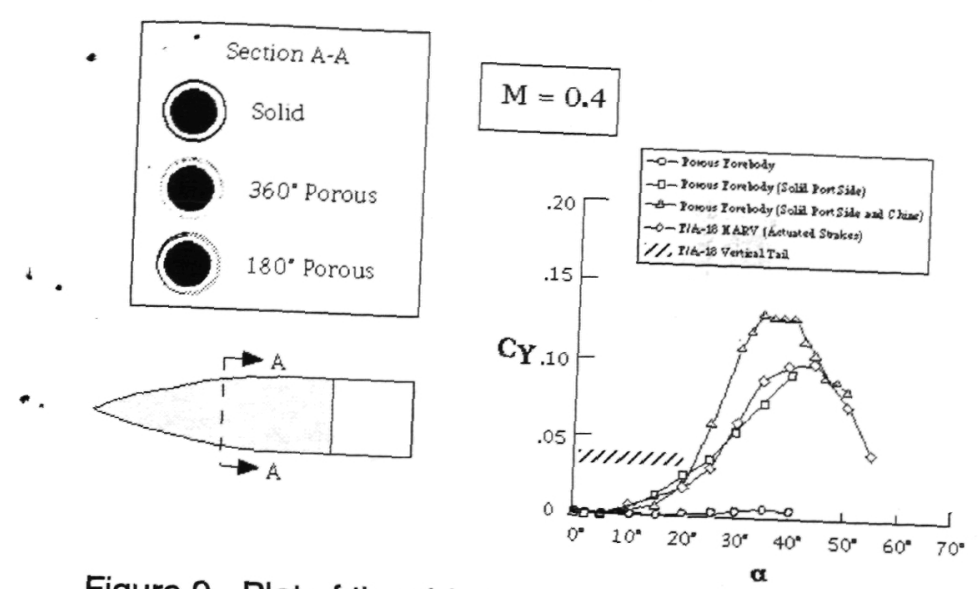

Figure 9. Plot of the side force coefficient for various circumferential extent of passive porosity on a 5 caliber, tangent-ogive model

\section{Flow Over a Hemispherical Ridge}

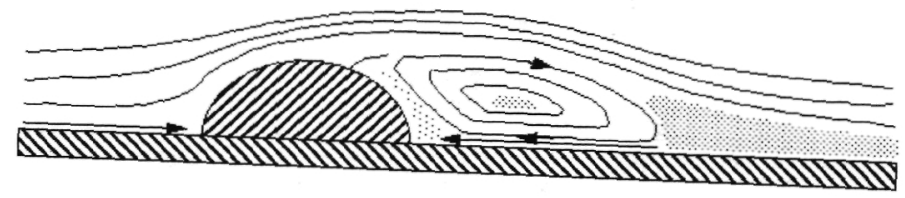

\section{Flow Over a Vertical Plate or Rectangular Ridge}

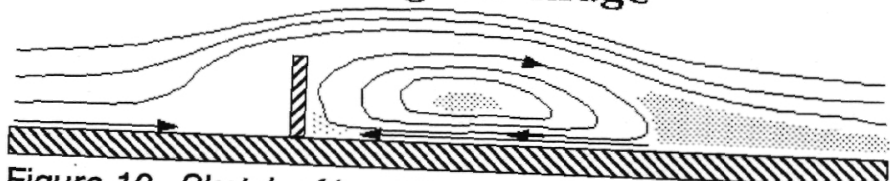

Figure 10. Sketch of local flow field charactictivit micro drag generators

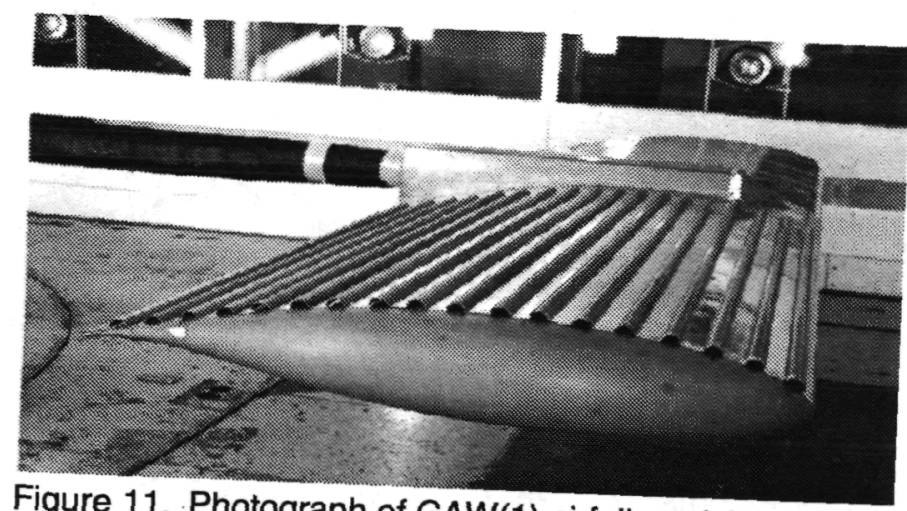

Figure 11. Photograph of GAW(1) airfoil model with large micro drag bumps installed.

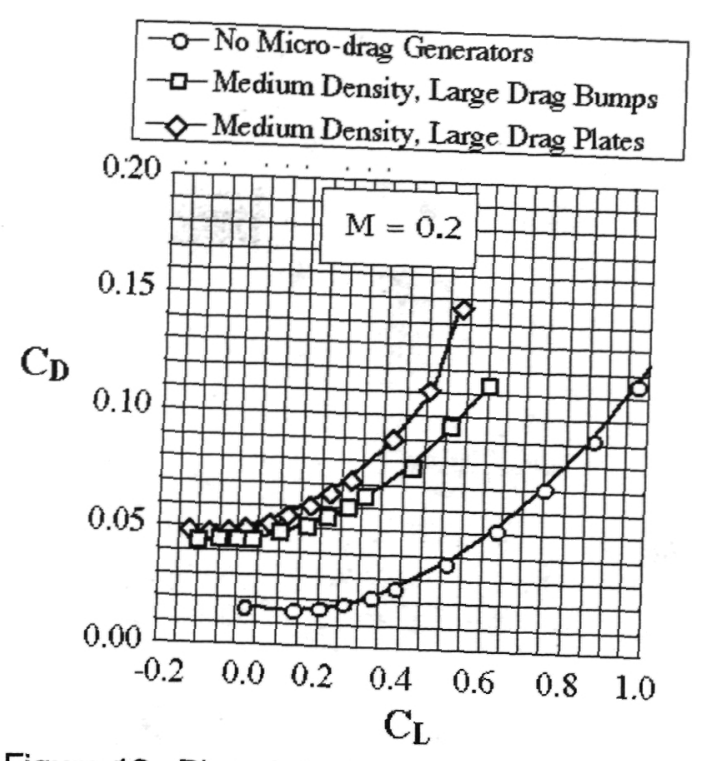

Figure 12. Plot of drag coefficient against lift coefficient for the GAW(1) wing with and without micro drag generators installed

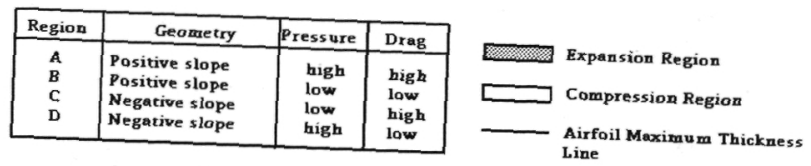

Upper Surface

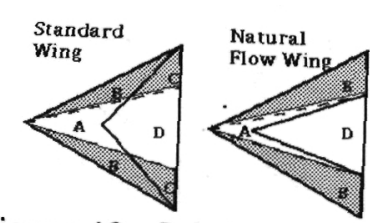

Figure 13. Graphic depicting Natural Flow design concept applied to a delta wing.

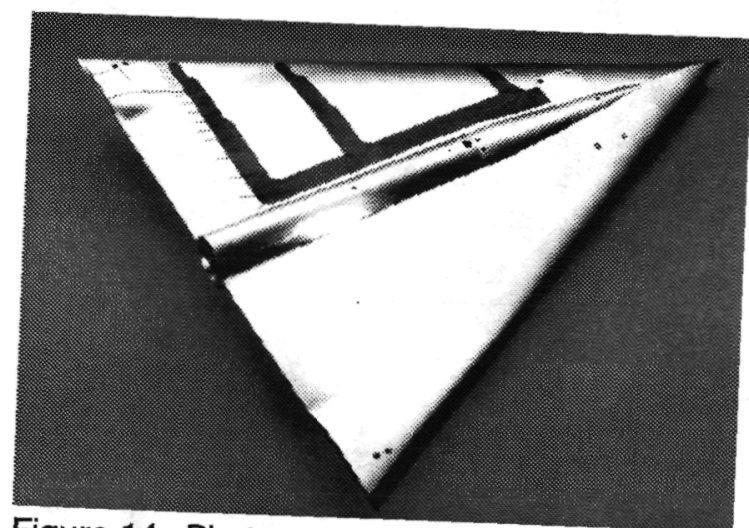
Figure 14. Photograph of Natural Flow $65^{\circ}$ delta wing
model. 


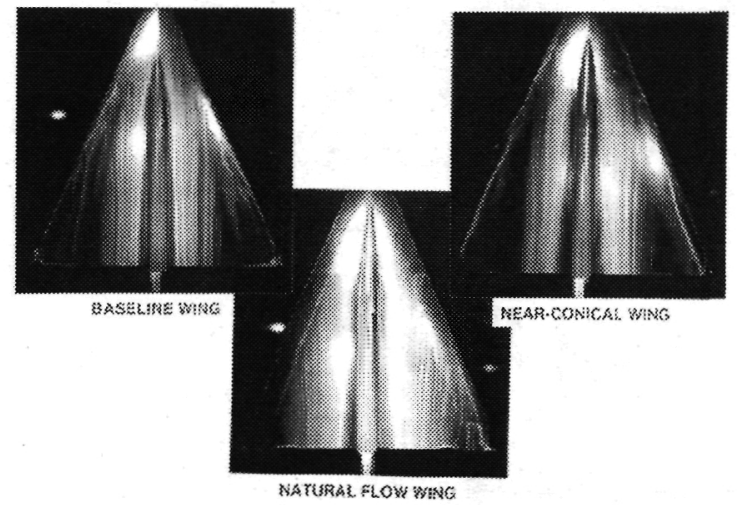

Figure 15. Oil-flow photographs of baseline, near conical, and natural flow $65^{\circ}$ delta wing models for $M=1.6$ and a lift coefficient of 0.3 .

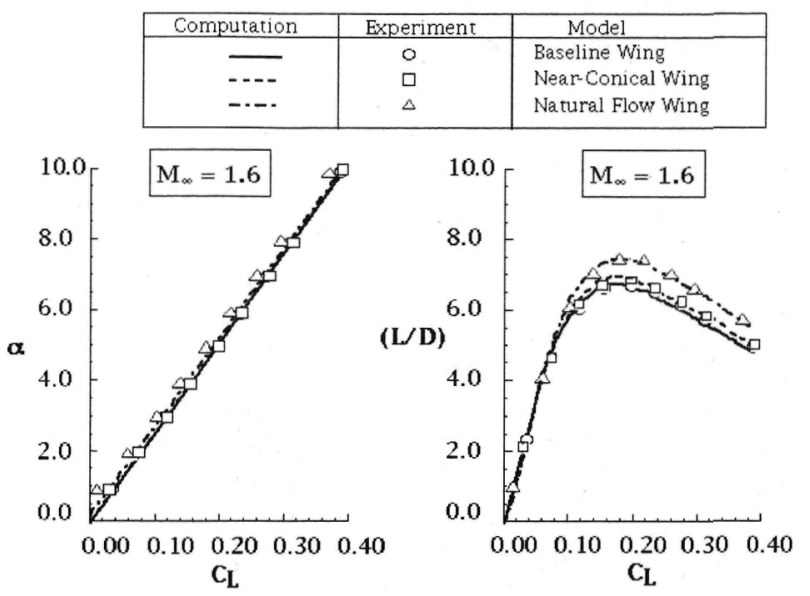

Figure 16. Plot of lift coefficient and lift-to-drag ratio for the baseline, near conical, and natural flow $65^{\circ}$ delta wing models.

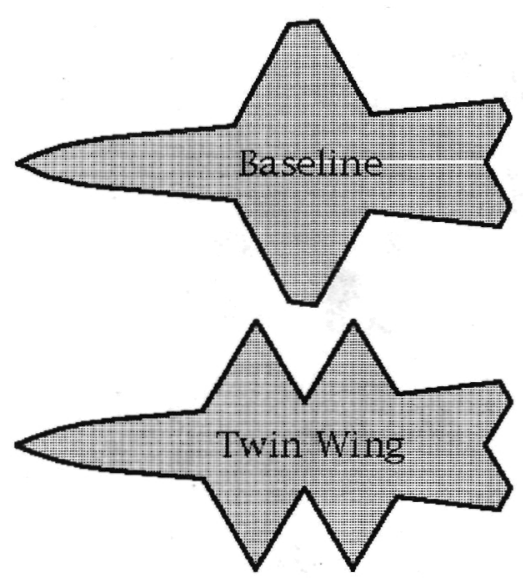

Figure 17. Sketch of baseline and twin wing advanced aircraft planform concepts.

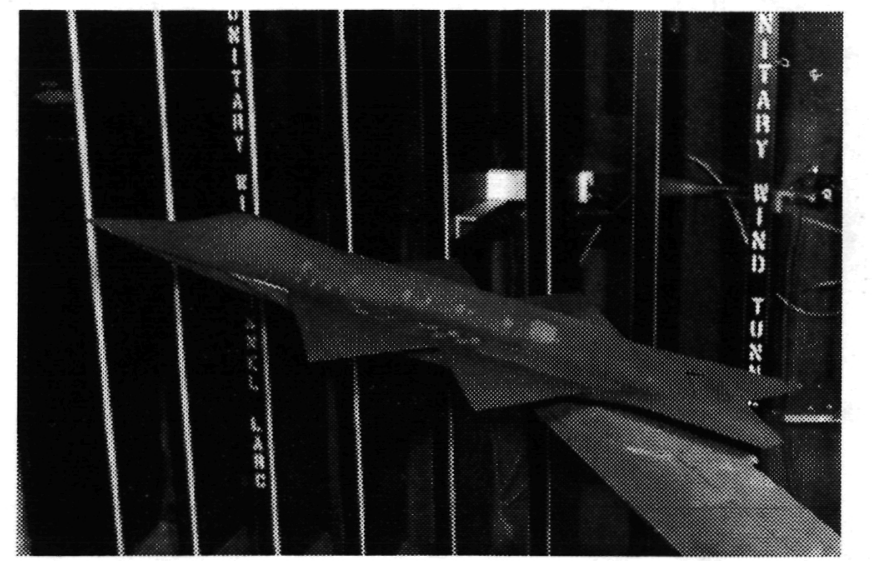

Figure 18. Photograph of the twin wing advanced aircraft concept model.

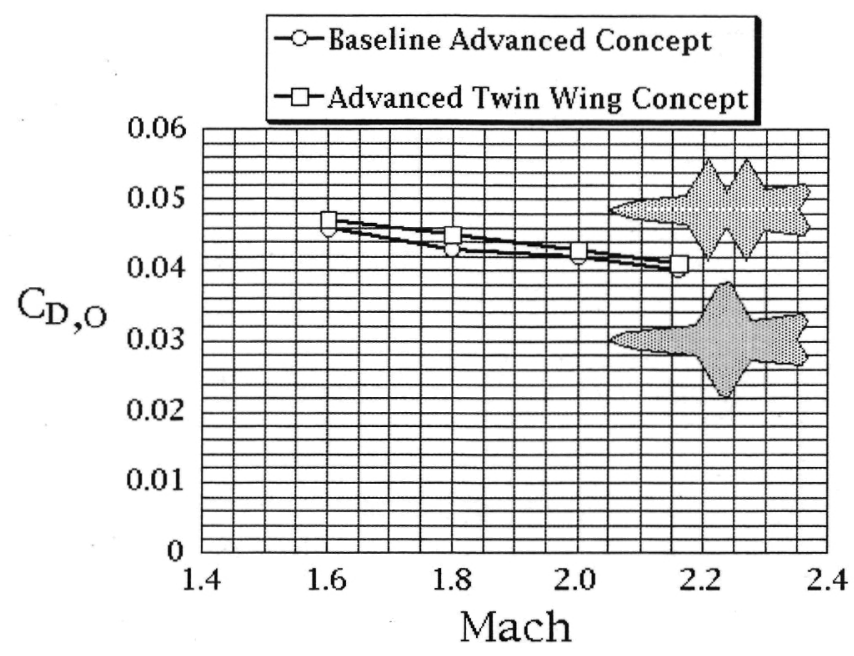

Figure 19. Plot of the zero-lift drag characteristics against Mach number for the baseline and twin wing advanced aircraft concepts.

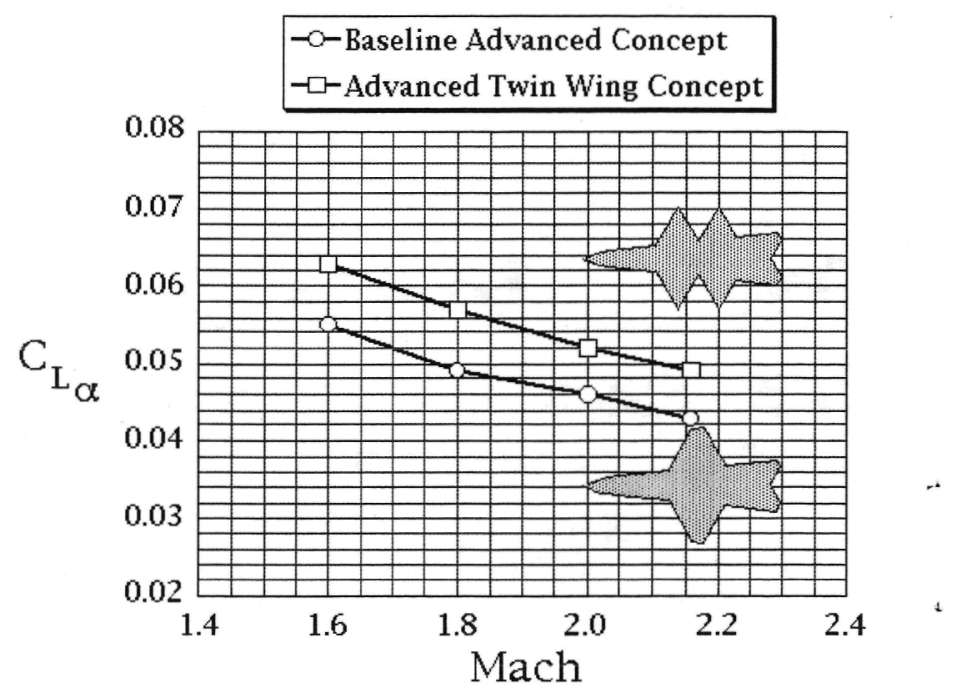

Figure 20. Plot of the lift-curve slope characteristics against Mach number for the baseline and twin wing advanced aircraft concepts. 